\title{
Visiones desiguales sobre la conservación en la periferia urbana: ganadores y perdedores del suelo de conservación en la Ciudad de México
}

\section{Contrasting Views on Conservation in the Urban Periphery: Winners and Losers of Conservation Areas in Mexico City}

Jorge Alberto Escandón Calderón ${ }^{I}$

\section{Resumen}

Este estudio se ubica en la Ciudad de México. El problema de investigación de este trabajo parte de que, en la periferia urbana de esta ciudad, las políticas de conservación tienen una expresión territorial interpretada de diversas maneras por algunos actores que están involucrados en su manejo. Se llevaron a cabo una serie de entrevistas en dos grupos focales: propietarios de la tierra y funcionarios públicos. Entre los resultados relevantes de este estudio se detectó que para el grupo de ejidatarios y comuneros, ${ }^{1}$ el principal problema es la falta de consulta a los dueños de la tierra en el territorio denominado suelo de conservación; mientras que para los funcionarios el principal problema es la falta de políticas que compitan con la especulación inmobiliaria y la venta de terrenos. Mientras los comuneros están observando las problemáticas en términos de su relación con las autoridades y desde una lógica territorial enfocada al entorno local, para la mayoría de los funcionarios éstas son de carácter institucional (falta de políticas; instrumentos de política rebasados; falta de coordinación) y se expresan a una escala territorial que tiende a omitir a los habitantes de los pueblos originarios.

Palabras clave: análisis de discurso; ejidatarios y comuneros; funcionarios; periferia urbana; suelo de conservación;

\footnotetext{
I Doctorado en Ciencias Políticas y Sociales por la Universidad Nacional Autónoma de México (UNAM), México. Investigador adscrito al Programa de Investigación en Cambio Climático de la Universidad Nacional Autónoma de México (UNAM), México. Líneas de interés: periferia urbana, conservación, ecología política, cambio climático. Correo electrónico: jescandon@ciencias.unam.mx

${ }^{1}$ Ejidatarios y comuneros son los propietarios de tierras que deciden los diferentes usos de la tierra a través de asambleas. El ejido es una forma ligeramente restringida de propiedad colectiva de la tierra, diseñada originalmente para beneficiar a los campesinos y estimular la producción agrícola, pero las reformas de la ley respectiva (1992) lo han hecho relativamente similar a una forma directa de propiedad.
} 


\begin{abstract}
This study is set in Mexico City. The research problem of this article begins with the premise that in the urban periphery, conservation policies have a territorial expression interpreted in different ways by some of the actors involved in its management. A series of interviews were conducted with two focus groups: landowners and public officials. Among the key results of this study, in the group of ejidatarios and comuneros, the main problem is the lack of consultation with the owners of the land in the territory called conservation areas. Whereas for public officials the main problem is the absence of policies able to compete with speculation and land selling. Whereas landowners see the problem in terms of their relationship with the authorities and from a territorial logic focused on the local environment, for most officials the difficulties are institutional (lack of policies, overwhelmed juridical instruments, lack of coordination) and are expressed on a scale that tends to omit the inhabitants of indigenous villages.
\end{abstract}

Keywords: urban periphery; conservation area; discourse analysis; landowners; public officials;

\title{
Introducción
}

Ante el embate de la urbanización acelerada a nivel mundial, hay que reconocer que este proceso es el principal impulsor de muchos de los cambios ambientales que están ocurriendo. En la actualidad, se estima que aproximadamente el 52 \% de la población mundial, o 3.6 mil millones, vive en áreas urbanas (UN DESA, 2013). Las zonas urbanas representan entre el $71 \%$ y el 76 \% de las emisiones de $\mathrm{CO} 2$ procedentes del uso final de la energía global, y entre el $67 \%$ y el $76 \%$ del consumo energético mundial (Seto et al., 2014). Para comprender cómo se hacen habitables las ciudades, es necesario observar también la contribución de la periferia urbana (Haase et al., 2012; Depietri et al., 2013; Baró et al., 2014; Larondelle et al., 2014). Los ecosistemas regionales periurbanos son cruciales para la salud y la habitabilidad de las ciudades, lo cual es una preocupación importante para el bienestar humano global, ya que cada vez más personas viven en áreas urbanas densamente construidas (MEA, 2005; Elmqvist et al., 2013; UN DESA, 2014).

En todo el mundo, las periferias urbanas han cambiado drásticamente en los últimos 40 años (Simon, 2008). Esas transformaciones son el resultado de múltiples factores, incluidas las actividades económicas, vinculadas tanto a los mercados de la tierra y la vivienda como a las estrategias de planificación urbana, rural y ambiental de los gobiernos locales y nacionales (Fisher, 


\section{Socjedad \\ Ambiente}

2003; Simon, 2008). Aunque existen diferencias significativas entre los países y dentro de ellos, también hay aspectos comunes que han proporcionado nuevos conocimientos sobre el estudio de las periferias, incluidos los siguientes: expansión urbana y cambio en el uso de la tierra (Nechyba y Walsh, 2004) así como el nuevo enfoque político en la gestión ambiental (Allen, 2003), para el cual los servicios ecosistémicos son centrales (Vejre et al., 2011).

La periferia urbana (a veces también llamada el margen urbano) puede considerarse como la forma urbana dominante y el desafío de planificación espacial del presente siglo. En la mayoría de los países en desarrollo, la periferia urbana es a menudo una zona de urbanización caótica con una creciente expansión. Puede verse como una franja entre la ciudad y el campo, o como un nuevo tipo de territorio multifuncional (Ravetz et al., 2013). Enfocarse en los procesos desiguales inherentes a la producción de los espacios urbanos, sirve como catalizador para una mejor comprensión de la dinámica de la periferia urbana, ya que el desarrollo periurbano se negocia a escala de la política local, enfrentando a una variedad de personas con diferentes puntos de vista que están en tensión y que impugnan el paisaje local.

Desde un punto de vista urbano clásico, la función principal de los espacios periurbanos es servir como reservas de suelo para la futura urbanización (Pérez-Campuzano et al., 2016). Sin embargo, otras perspectivas (como la planificación para el desarrollo y el desarrollo sustentable) enfatizan la importancia de los servicios ambientales brindados por dichos espacios a la ciudad (Portnov y Pearlmutter, 1999; Allen, 2003; Da Gamma Torres, 2008; Hornis y Eck, 2008; Huang et al., 2009; Kritsanaphan y Sajor, 2011).

Desde una perspectiva geográfica, el estudio del área que conforma la periferia urbana de las grandes ciudades, implica tratar con un territorio que expresa una situación de interfaz entre dos formas territoriales aparentemente bien diferenciadas: el campo y la ciudad (Keil y Macdonald, 2016). El límite territorial entre los cinturones verdes y los suburbios ubicados en la periferia urbana, separa y negocia un conjunto cada vez más complejo de socionaturalezas producidas, que desmienten la simplicidad original de las dicotomías ciudad-campo característicos de los primeros imaginarios asociados con los cinturones verdes (Keil y Macdonald, 2016) ubicados en la periferia urbana. Con respecto a la periferia urbana, la "teoría metropolitana" ha sido una forma aceptada de analizar los patrones de estructura de los asentamientos urbanos y ha producido resultados útiles, e interesantes. Sin embargo, Taylor (2011) sostiene que, a pesar de todo este trabajo, no se debe olvidar que la periferia urbana además de ser una zona geográfica, también es un área donde confluyen diversas formas de vida con múltiples interconexiones sociales, económicas, políticas y ambientales.

Sociedad y Ambiente, 23, 2020, ISSN: 2007-6576, pp. 1-29. doi: 10.31840/sya.vi23.2149 


\section{Suelo de conservación de la Ciudad de México, como parte de la periferia urbana}

La Ciudad de México ${ }^{2}$ abarca un área de aproximadamente 148 mil km2 con una población de más de 8.5 millones, lo que hace que sea la ciudad más densamente poblada de México. La Ciudad de México está dividida en dos grandes áreas: la urbana y la de conservación. La primera corresponde a la zona de construcción (la infraestructura urbana), y la última es una categoría administrativa diseñada para proteger los recursos naturales y ambientales. El suelo de conservación (SC) de la Ciudad de México es un área designada por la ley en el ordenamiento territorial; en el "Programa General de Ordenamiento Ecológico del Territorio" (PGOETDF, 2000), el SC representa el 58 \% de la superficie total de la Ciudad de México (85 mil ha) (Figura 1).

Figura 1. Compuesto de falso color de imagen satelital de la Ciudad de México, con la delimitación del suelo de conservación (línea roja)

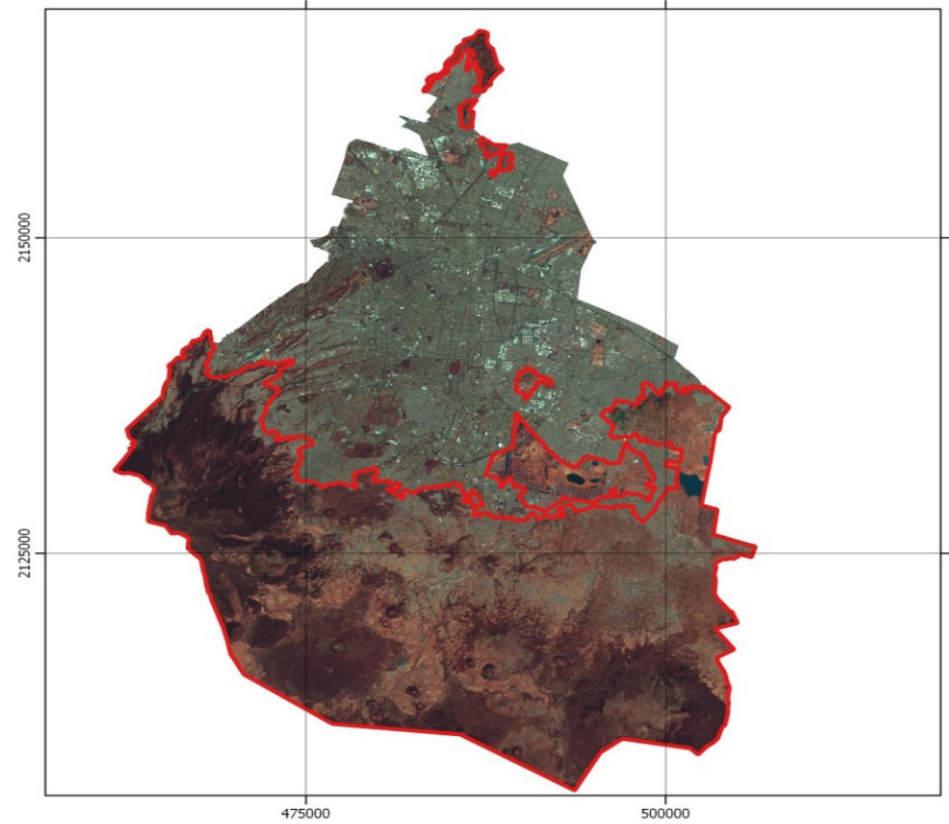

Fuente: elaboración propia a partir de imagen LANDSAT. IMAGEN SATELITAL (2017) DE LA CIUDAD DE
MÉXICO
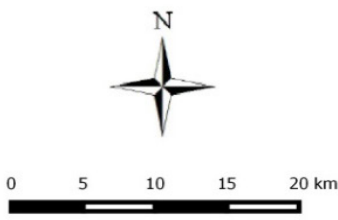

Los bosques y la agricultura del SC son los usos del suelo primarios y estos usos son también los mayores contribuyentes a los servicios ecosistémicos como resultado de las características del suelo y el bosque (Aguilar y Santos, 2011a, 2011b; Saavedra et al., 2011). La zona de conservación

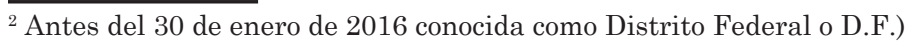


infiltra cerca del $70 \%$ de toda el agua que se consume en la Ciudad de México (Perevochtchikova y Vázquez, 2012); 90 toneladas/ha/año de dióxido de carbono es capturado por su bosque, 18 mil especies de plantas y animales viven en la zona y es también un sitio que brinda servicios recreativos y paisajísticos (Castelán y Mejía, 2011).

\section{Dimensión social del suelo de conservación}

Es importante tener en cuenta que la propiedad de la tierra en el SC es principalmente colectiva (comunidades y ejidos). Más del $80 \%$ de la superficie boscosa (38 252 ha de coníferas y latifoliadas) del SC es de propiedad social. Son tres los distritos administrativos que concentran la mayor parte de la zona de conservación: la delegación de Milpa Alta (32.2 \%), Tlalpan (29.4 \%) y Xochimilco (11.9 \%) (GDF, 2014). Dentro del SC viven alrededor de 850 mil personas, distribuidas en 36 núcleos poblacionales tanto en asentamientos humanos regulares como irregulares; de los cuales 29 son ejidos y siete son comunidades (PAOT, 2009). En este territorio se realizan las actividades de agricultura y ganadería de la Ciudad de México. Dado que existe una veda forestal desde 1946 las actividades silvícolas legales que se realizan son sólo las de saneamiento forestal.

En la primera década de este siglo, la población económicamente activa ocupada en actividades primarias era de 20600 personas, lo que representa el 0.6 \% total de la población económicamente activa ocupada por actividad económica en la entidad (INEGI, 2006, 2010). A pesar de las reducidas dimensiones de la actividad agropecuaria, algunos cultivos son significativos en la economía nacional. Esto es el caso del nopal que se produce principalmente en Milpa Alta, del romerito en Tláhuac y de la flor de Nochebuena en Xochimilco (Sheinbaum, 2011). Sin embargo, el producto de la actividad agropecuaria representa sólo una pequeña parte de los ingresos de las familias que poseen tierras en el SC. La cercanía con la ciudad y el incremento en la educación ha llevado a los hijos y nietos de ejidatarios y comuneros ocuparse en actividades del sector secundario y, sobre todo, del terciario (Sheinbaum, 2011).

Los barrios y comunidades ubicadas en el SC o en lo que correspondería a la periferia de la Ciudad de México, sobresalen por la mayor presencia en sus unidades territoriales de los grupos de bajo y muy bajo desarrollo. Las peores condiciones de educación, salud y vivienda por manzana reflejan un patrón espacial que se relaciona con varios factores, como son: los menores ingresos, el menor costo del suelo, la localización de asentamientos irregulares en zonas de reserva o de mayor riesgo, así como las dificultades de accesibilidad reflejada en la falta de equipamiento y servicios urbanos. Si se toman en cuenta a todas las delegaciones que tienen territorio en el SC, en dichas entidades administrativas el índice de desarrollo social está catalogado entre muy bajo y bajo (Evalúa D.F., 2011). 
En las últimas décadas este territorio ha enfrentado intensos cambios de uso del suelo (urbanización) (Pérez et al., 2011, 2012) que son principalmente el resultado de los asentamientos irregulares (Pérez Campuzano et al., 2016). Aguilar (2008) menciona con respecto a la población del área metropolitana de la Ciudad de México, que el crecimiento demográfico ha mantenido un ritmo lento, a una tasa del $0.89 \%$ para toda la ciudad. Sin embargo, lo que corresponde propiamente al Distrito Federal ha tenido una tasa de crecimiento de $0.27 \%$, mientras que el resto de la periferia metropolitana, correspondiente a los estados de México e Hidalgo, tuvo una tasa de crecimiento de $1.43 \%$ a principios del siglo XXI. Gran parte del crecimiento periférico está relacionado con barrios pobres y asentamientos informales que se desarrollan en estas áreas; un proceso que ocurre incluso en el SC, a una tasa de crecimiento que duplica la de toda la entidad (Aguilar, 2008).

\section{Los discursos como aproximación a la naturaleza}

En el agresivo proceso de urbanización de la naturaleza, que define el proceso de crecimiento urbano actual (Keil, 2013), el límite territorial de la periferia urbana separa y negocia un conjunto cada vez más complejo de producciones de socionaturalezas que desmienten la simplicidad original de las dicotomías urbano-rurales en los primeros imaginarios asociados con los cinturones verdes (Keil y Macdonald, 2016).

Siguiendo, por un lado, las ideas de Latour (1993, 1998, 2004), quien rechaza contundentemente la noción de la naturaleza como algo "puro" e introduce el concepto de "híbridos", definiéndolos como "la mezcla de la naturaleza y la cultura" (1993: 10), y por otro, las de Swyngedouw (2006), quien profundizando afirma que los espacios híbridos están formados por una variedad de procesos "naturales" - tanto biológicos, físicos y químicos—, por prácticas materiales, culturales y discursivas de diversos actores, así como por las relaciones sociales generadas entre los actores, deducimos que los espacios híbridos tienen tres dimensiones: una dimensión material física única; una dimensión práctica y mercantilizada; así como una dimensión construida discursivamente (Zimmer, 2010).

Uno de los principales puntos a aclarar dentro del concepto de hibridación es la manera de detectar los conflictos dentro de los espacios híbridos a partir del análisis del discurso dentro del cual éstos se encuentran incrustados. También comporta una gran importancia determinar quiénes son los ganadores y perdedores de las formas específicas de hibridación. Este hecho apunta a la importancia de la diversidad en los entornos urbanos, lo cual no se ha reconocido suficientemente. Las relaciones sociales con la naturaleza y las relaciones sociales en sí se refuerzan de esta manera: por un lado, la posición social de un actor se fortalece mediante la realización de prácticas ambientales percibidas como legítimas; pero al mismo tiempo, la legitimidad de ciertas formas de interacción con sistemas no humanos puede ponerse a discusión o bajo cuestionamiento. Existe

Sociedad y Ambiente, 23, 2020, ISSN: 2007-6576, pp. 1-29. doi: 10.31840/sya.vi23.2149 
además un vínculo de esta legitimidad con discursos ambientales o discursos sociales más amplios, como aquellos relacionados con procesos de control y disciplina (Oliver, 2006). Por otra parte, ciertas prácticas se hacen inteligibles a través de las instituciones (en el sentido de las reglas y normas) que les dan legitimidad; sobre todo indagando en la participación democrática en las instituciones que influyen en la producción y la gestión de las relaciones sociales con la naturaleza.

Esta investigación se basa en un marco de ecología política, que analiza cómo los discursos competitivos y las relaciones de poder afectan el uso de la tierra y la gestión de los recursos naturales, generando ganadores y perdedores (Escobar, 1996; Robbins, 2004). Los discursos se definen como "redes de significados, ideas, interacciones y prácticas que se expresan o representan en textos (lenguaje hablado y escrito, gestos e imágenes visuales), dentro de entornos institucionales y cotidianos" (Bischoping y Gazso, 2016: 1). Estos discursos están formados por las relaciones sociales, las relaciones de poder y las instituciones, las cuales a su vez tienen un impacto en las relaciones sociales (Buchanan, 2013; Hajer, 1995; Rikoon, 2006). Comprender este nexo de discursos y relaciones sociales es fundamental para analizar las construcciones sociales y las negociaciones en torno al manejo de los recursos naturales (Castree y Braun, 2001).

En el marco adoptado se identifican las interdependencias sociales clave del sistema y se las analiza de acuerdo con cuatro dimensiones: 1) el grado de conciencia de las partes interesadas sobre las interdependencias; 2) las instituciones formales e informales que regulan estas interdependencias; 3) los niveles de organización en los que operan los actores, y 4) las relaciones de poder que los afectan (Barnaud et al., 2018). Tanto Hajer (1995) como Foucault (1970) asumen el poder como una fuerza que recorre la totalidad del campo social, tanto estructurando a los sujetos, como posibilitando el control del discurso en la producción de significados. La ecología política proporciona el marco teórico para pensar sobre las relaciones de poder y la confrontación discursiva en torno a estas categorías.

\section{Marco metodológico}

El objetivo general de este estudio es analizar cómo ejidatarios y comuneros, así como autoridades gubernamentales de la Secretaría del Medio Ambiente, de la Secretaría de Desarrollo Rural y de la Dirección de Ecología de la Delegación Tlalpan, construyen la problemática ambiental del SC, así como sus posibles soluciones. El propósito es descubrir los elementos involucrados en las negociaciones entre ejidatarios y comuneros propietarios de la superficie donde se ubica el SC y las autoridades gubernamentales cuya responsabilidad es la de preservar los recursos naturales.

Crowe et al. (2011: 1) afirman: "Un estudio de caso es un enfoque de investigación que se utiliza para generar una comprensión profunda y multifacética de un tema complejo en su contexto 
de la vida real". Nuestro desafío fue diseñar un método que capturara el momento. Un principio fundamental de nuestro diseño de investigación fue reconocer el estado marginal de "falta de voz" o invisibilización de las familias de agricultores cuyas prácticas se centran en no usar agroquímicos, desafiando así "el monopolio que otros grupos de productores han establecido sobre la producción de conocimiento" (Kirby y McKenna, 1989: 65).

Identificamos tres supuestos clave para respaldar la investigación: primero, que a medida que el conocimiento se construye socialmente, también la experiencia sigue este proceso. Segundo, debido a que las interacciones sociales forman la base del conocimiento social, para desarrollar narrativas de experiencia se hace necesaria una forma más íntima de entender el contexto social dentro del cual viven las personas. En tercer lugar, que los agricultores que no utilizan agroquímicos construyen el SC de manera diferente, no sólo por sus roles y responsabilidades particulares, sino también por sus propias perspectivas sobre el mundo que los rodea.

Los métodos utilizados para recabar información de nuestros encuestados fueron: discusiones focales de grupos, entrevistas semiestructuradas y diseño de estudio de caso. Los grupos focales se crearon con productores masculinos y femeninos, así como grupos combinados que incluían a otras partes interesadas, como extensionistas y funcionarios ambientales. Para generar los grupos focales de funcionarios existieron desafíos de reclutamiento; muchos de los intentos fracasaron debido a la desconfianza de los funcionarios para otorgar entrevistas, lo cual determinó el tamaño de la muestra. Para el grupo de productores que no usan agroquímicos, primero entrevistamos a todos los que nos brindaban su tiempo y luego, conforme fuimos construyendo una relación de confianza en las distintas comunidades, comenzamos a identificar las redes y relaciones entre las diversas familias conectadas a través de su experiencia de manejo de terrenos agrícolas sin agroquímicos en el SC.

Para seleccionar a los participantes se combinó una estrategia de muestreo dirigido con el muestreo de "bola de nieve". Este último método de muestreo se utilizó debido a que entre sus premisas considera que la muestra cobra validez en el momento en que un conjunto de respuestas de las personas entrevistadas, empieza a ser "repetitiva". Dada la perspectiva temporal del proyecto, los funcionarios participantes se seleccionaron con base en su experiencia en la región: individuos con experiencia en la política local regional y estatal de conservación, así como representantes de los productores agrícolas cuyos métodos se caracterizaban por no utilizar agroquímicos en sus cultivos. Pedimos a los participantes recomendar a otros que cumplieran con los criterios de selección. Todas las entrevistas se hicieron de forma individual.

Los grupos focales se utilizaron para desarrollar el cuestionario detallado, y la discusión se centró en torno a las siguientes preguntas: 1) ¿Cuáles son los diferentes relatos sobre el SC en la Ciudad de México?, y 2) ¿Cómo se visualizan los problemas y las consecuencias en las narrativas 
sobre el SC y el desarrollo de políticas en la periferia (borde) urbana? Se realizaron también entrevistas semiestructuradas con aquellos interesados que accedieron a participar, de los cuales quince eran comuneros y otros quince eran funcionarios de la Secretaría del Medio Ambiente del Distrito Federal, de la Secretaría de Desarrollo Rural del Distrito Federal y de la delegación Tlalpan (Cuadro 1). Así, las entrevistas consistieron en una serie de preguntas abiertas, así como preguntas detalladas, en las que se quiso indagar acerca de las percepciones sobre las políticas de conservación y desarrollo rural, con relación a la efectividad para contrarrestar la expansión urbana.

Cuadro 1. Informantes entrevistados

\begin{tabular}{|l|l|c|}
\hline \multicolumn{1}{|c|}{ Informantes clave } & \multicolumn{1}{|c|}{ Adscripción } & Número de entrevistados \\
\hline \multirow{4}{*}{ Funcionarios } & Secretaría del Medio Ambiente GDF & 5 \\
\cline { 2 - 3 } & Delegación Tlalpan & 5 \\
\cline { 2 - 3 } & Secretaría de Desarrollo Rural GDF & 5 \\
\hline \multirow{4}{*}{ Ejidatarios y comuneros } & San Andrés Totoltepec & \multicolumn{2}{|c|}{5} \\
\cline { 2 - 3 } & San Miguel Topilejo & 3 \\
\cline { 2 - 3 } & Magdalena Petlacalco & 3 \\
\cline { 2 - 3 } & San Miguel y Santo Tomás Ajusco & 2 \\
\cline { 2 - 3 } & San Miguel Xicalco & 2 \\
\hline Total & & 30 \\
\hline
\end{tabular}

Fuente: elaboración propia a partir de datos obtenidos del trabajo de campo.

Se organizaron las transcripciones de las entrevistas en una base de datos y se analizaron con el apoyo del programa de análisis cualitativo de datos ATLAS.ti (Universidad Técnica de Berlín, Alemania). Se utilizó un enfoque de codificación inductivo para identificar los temas relacionados con el papel del SC en el desarrollo de las actividades de los comuneros, así como en el desempeño de los funcionarios. En primer lugar, se realizó la lectura de las transcripciones varias veces para identificar temas generales relacionados con nuestras preguntas rectoras. Inicialmente se codificaron los temas generales. Después, dentro de cada tema general se subcodificaron los diferentes puntos de vista identificados sobre el tema, tomando nota del lenguaje que describe las características de los problemas del SC, la agricultura, las tendencias del desarrollo urbano, las soluciones a futuro y las contribuciones que se esperan para el desarrollo regional.

Nuestra intención era captar la diversidad de narrativas entre las entrevistas. El problema de investigación explora diferentes enfoques y narrativas sobre el futuro de la conservación en la 
periferia urbana, así como la gestión de recursos naturales desde la política local, comparando la experiencia entre lo que dicen los funcionarios y los propietarios de la tierra. Analizar las políticas de conservación en la periferia urbana es interesante por la manera en que se construyen los discursos acerca de los beneficios que esos territorios aportan en términos de bienes y servicios ambientales a la ciudad. Se complementó y contextualizó el análisis de las entrevistas con información de documentos de política, artículos de prensa y literatura académica.

\section{Resultados}

\section{¿Cómo se comprende y define el suelo de conservación?}

Un acercamiento inicial muestra diferencias entre lo que comprenden los funcionarios y lo que comprenden los propietarios en cuanto al SC (Cuadro 2).

\section{Cuadro 2. Opiniones sobre cómo se comprende y define el suelo de conservación (SC)}

\begin{tabular}{|c|c|c|}
\hline \multirow{2}{*}{ Tema } & \multicolumn{2}{|l|}{ Respuestas } \\
\hline & Ejidatarios y comuneros & Funcionarios \\
\hline $\begin{array}{l}\text { ¿Cómo se } \\
\text { comprende } \\
\text { y define el } \\
\text { SC? }\end{array}$ & $\begin{array}{l}\text { - El SC constituye una medida } \\
\text { para la preservación de áreas } \\
\text { boscosas y ciclos naturales. } \\
\text { - El SC para sus habitantes, tan- } \\
\text { to comuneros como ejidatarios, } \\
\text { significa la posibilidad de desa- } \\
\text { rrollar actividades agrícolas y } \\
\text { pecuarias que constituyen una } \\
\text { parte importante de su propia } \\
\text { cultura e identidad. } \\
\text { - Los habitantes del SC tienen } \\
\text { claro que sus ejidos y comu- } \\
\text { nidades forman parte o están } \\
\text { comprendidos dentro del SC, } \\
\text { pero destacan que existe impre- } \\
\text { cisión y que los límites del SC } \\
\text { no están definidos claramente, } \\
\text { ocasionando confusiones. }\end{array}$ & $\begin{array}{l}\text { - De acuerdo con los funcionarios, el establecimiento } \\
\text { del SC tiene que ver con la preservación de los rasgos } \\
\text { "naturales" de un espacio, con zonas intocadas que se } \\
\text { conservan contribuyendo a la sustentabilidad de la ciu- } \\
\text { dad. } \\
\text { - Destacan el carácter normativo y jurídico del SC y su } \\
\text { importancia como una medida para reglamentar el } \\
\text { uso del territorio. Es una categoría legal o jurídica im- } \\
\text { plementada por el gobierno de la ciudad para limitar } \\
\text { la expansión urbana y procurar que las áreas protegi- } \\
\text { das no se vean afectadas por el cambio de uso de suelo. } \\
\text { - Los funcionarios entrevistados, aunque reconocen la } \\
\text { existencia física del SC, sus rasgos ecológicos o bioló- } \\
\text { gicos y la presencia de comunidades, ejidos y asenta- } \\
\text { mientos irregulares dentro de la demarcación de los } \\
\text { mismos, al igual que los pobladores del SC, no pueden } \\
\text { definir y mencionar con claridad los límites de esta } \\
\text { zona de protección. }\end{array}$ \\
\hline
\end{tabular}

Fuente: elaboración propia a partir del análisis de entrevistas. 
Para todos los funcionarios, el SC es una categoría legal o jurídica implementada por el gobierno de la Ciudad para limitar la expansión urbana y procurar que las áreas protegidas no se vean afectadas por el cambio de uso de suelo, la urbanización, así como preservar dicho territorio en su estado "natural". Para 80 \% de los funcionarios, el SC es un espacio o superficie que da sustentabilidad ambiental a la ciudad, con una clasificación que norma y dictamina una serie de actividades y establece restricciones para el uso urbano. Para todos los comuneros el SC es un territorio para proveer de recursos y para preservar aspectos culturales. Desde su comprensión, el SC es una porción de la ciudad con atributos muy diferentes al resto del espacio urbano y estos rasgos deben mantenerse. Para $87 \%$ de los comuneros y ejidatarios entrevistados, destaca la idea de que el SC constituye una medida para la preservación de áreas boscosas y ciclos naturales.

\section{Sobre los problemas ambientales más graves detectados en el suelo de conservación}

Todos los funcionarios mencionan que la degradación del SC es por efecto de la urbanización. De los funcionarios, 80 \% relacionan la degradación del SC con la extracción de recursos y con la falta de capacidad gubernamental. Un $50 \%$ de los funcionarios mencionan la falta de certeza en la tenencia de la tierra y la restricción en los usos del suelo para los propietarios (Cuadro 3).

\section{Cuadro 3. Opiniones sobre los problemas ambientales más graves del suelo de conservación (SC)}

\begin{tabular}{|c|c|c|}
\hline \multirow{2}{*}{ Opiniones } & \multicolumn{2}{|l|}{ Respuestas } \\
\hline & Ejidatarios y comuneros & Funcionarios \\
\hline $\begin{array}{l}\text { Sobre los } \\
\text { problemas } \\
\text { ambientales más } \\
\text { graves detectados } \\
\text { en el SC }\end{array}$ & $\begin{array}{l}\text { - La falta de agua } \\
\text { - La expansión urbana } \\
\text { - La disminución de bosques } \\
\text { - La contaminación del aire por el uso de } \\
\text { vehículos } \\
\text { - La falta de educación ambiental } \\
\text { - La utilización de agroquímicos como con- } \\
\text { taminantes del suelo }\end{array}$ & $\begin{array}{l}\text { - Pérdida por urbanización } \\
\text { - Pérdida por extracción de recursos } \\
\text { - Pérdida por falta de capacidad gu- } \\
\text { bernamental } \\
\text { - Falta de certeza en la tenencia de la } \\
\text { tierra } \\
\text { - Restricción en los usos de suelo para } \\
\text { los propietarios }\end{array}$ \\
\hline
\end{tabular}

Fuente: elaboración propia a partir del análisis de entrevistas. 
Todos los comuneros y ejidatarios perciben como problema ambiental más grave del SC la falta de agua. Un 90 \% de ellos mencionan además que el problema principal es la expansión urbana. Un 66 \% de los comuneros y ejidatarios mencionan la disminución de bosques y la contaminación del aire por autos. Más del 50 \% de las respuestas mencionan la falta de educación ambiental y la utilización de agroquímicos como contaminantes del suelo.

\section{Sobre las dificultades en el suelo de conservación}

Los funcionarios y comuneros priorizan de forma distinta las dificultades del SC (Cuadro 4). Cerca del $85 \%$ de los funcionarios mencionan como dificultad la falta de políticas agropecuarias y forestales que compitan con la especulación inmobiliaria y la venta de terrenos. Un $60 \%$ de los funcionarios identifica como dificultad la falta de incentivos económicos para la conservación que puedan traducirse en beneficios directos a ejidos y comunidades, así como la falta de coordinación entre distintas autoridades con atribución en SC. Sólo la mitad de los funcionarios menciona como una dificultad la falta de consenso con comuneros y ejidatarios.

\section{Cuadro 4. Opiniones sobre las dificultades del suelo de conservación (SC)}

\begin{tabular}{|c|c|c|}
\hline \multirow{2}{*}{ Tema } & \multicolumn{2}{|l|}{ Respuestas } \\
\hline & Ejidatarios y comuneros & Funcionarios \\
\hline $\begin{array}{l}\text { Sobre las } \\
\text { dificultades } \\
\text { del SC }\end{array}$ & $\begin{array}{l}\text { - Exclusión de consulta a las auto- } \\
\text { ridades comunitarias (asambleas } \\
\text { y presidente de bienes comunales) } \\
\text { de los pueblos originarios en las } \\
\text { políticas del SC } \\
\text { - Falta de comunicación entre auto- } \\
\text { ridades y comuneros } \\
\text { - Especulación inmobiliaria y venta } \\
\text { de terrenos } \\
\text { - Falta de planeación por parte de } \\
\text { las instancias de gobierno }\end{array}$ & $\begin{array}{l}\text { - Falta de políticas agropecuarias y forestales que } \\
\text { compitan con especulación inmobiliaria y venta } \\
\text { de terrenos } \\
\text { - Falta de incentivos económicos con beneficios di- } \\
\text { rectos para los ejidatarios y comuneros } \\
\text { - Falta de coordinación entre autoridades guberna- } \\
\text { mentales } \\
\text { - Falta de consenso con comuneros y ejidatarios }\end{array}$ \\
\hline
\end{tabular}

Fuente: elaboración propia a partir del análisis de entrevistas.

Para más del $90 \%$ de los comuneros, una de las grandes dificultades en la gestión del SC, es la falta de comunicación y de una consulta amplia con las comunidades, así como la ausencia de toma 
de acuerdos y decisiones consensuadas entre las comunidades y las autoridades. Entre el $65 \%$ y el $70 \%$ de los comuneros y ejidatarios ven la especulación inmobiliaria y la venta de terrenos como una de las dificultades del SC. Más del $50 \%$ de los comuneros entrevistados mencionaron que las autoridades son quienes toman las decisiones determinando qué y dónde se va a conservar, sin tener una comprensión cabal y un conocimiento detallado de lo que sucede en el SC.

\section{Sobre la expansión urbana como problema para comuneros y ejidatarios}

En cuanto a las narrativas relacionadas con la expansión urbana, funcionarios y comuneros atribuyen diversas causas (Cuadro 5). El 85 \% de las respuestas de los funcionarios ven como problema que los programas delegacionales de desarrollo urbano sean instrumentos para regularizar asentamientos humanos irregulares. Un $60 \%$ de los funcionarios también menciona incompatibilidad entre los programas de desarrollo urbano municipal y el programa general de ordenamiento ecológico, los cuales operan en conjunto en el SC. El $53 \%$ de los funcionarios mencionan la presencia de desarrolladores inmobiliarios con intereses económicos que fomentan la especulación y la venta de terrenos. El 40 \% de las respuestas de los funcionarios menciona la promoción por parte de grupos políticos de asentamientos humanos irregulares.

\section{Cuadro 5. Opiniones sobre la expansión urbana}

\begin{tabular}{|c|c|c|}
\hline \multirow{2}{*}{ Tema } & \multicolumn{2}{|l|}{ Respuestas } \\
\hline & Ejidatarios y comuneros & Funcionarios \\
\hline $\begin{array}{l}\text { Sobre la ex- } \\
\text { pansión ur- } \\
\text { bana como } \\
\text { problema }\end{array}$ & $\begin{array}{l}\text { - Violación a normas de ocupación } \\
\text { del suelo y falta de vigilancia por } \\
\text { parte de las autoridades guber- } \\
\text { namentales } \\
\text { - Falta de planeación } \\
\text { - Promoción de asentamientos hu- } \\
\text { manos irregulares por parte de } \\
\text { diputados y políticos }\end{array}$ & $\begin{array}{l}\text { - Los programas delegacionales de desarrollo urbano } \\
\text { como instrumentos para regularizar asentamientos } \\
\text { humanos } \\
\text { - Incompatibilidad entre criterios ambientales y ur- } \\
\text { banos del SC } \\
\text { - Presencia de desarrolladores inmobiliarios con in- } \\
\text { tereses económicos relacionados con especulación } \\
\text { inmobiliaria y venta de terrenos }\end{array}$ \\
\hline
\end{tabular}

Fuente: elaboración propia a partir del análisis de entrevistas.

Del grupo de comuneros y ejidatarios, 87 \% mencionan que la expansión urbana se debe a falta de planeación por parte de las autoridades gubernamentales. En las respuestas de los comuneros, 67 $\%$ mencionan la promoción de asentamientos humanos irregulares por parte de diputados y polí- 
ticos. La mitad de los comuneros y ejidatarios menciona como causa de la expansión, la migración de gente de otros estados y los avecindados, los cuales muchas veces violan la ley, sobre todo en predios indefinidos. Además, cerca del $45 \%$ de las respuestas mencionan a actores de niveles socioeconómicos altos que se presentan como compradores de terrenos.

\section{Sobre las políticas y programas gubernamentales}

Las opiniones sobre los programas y políticas gubernamentales tienen elementos de coincidencia entre propietarios y funcionarios (Cuadro 6). En la narrativa de los funcionarios, $86 \%$ de las respuestas perciben los programas de conservación como carentes de una visión integral y de estrategias de desarrollo rural. Igual porcentaje de respuestas mencionan la falta de recursos económicos y humanos, así como instrumentos legales atrasados y rebasados. Un $73 \%$ de los funcionarios mencionan la falta de continuidad de los programas que operan en el SC, poniendo de manifiesto la carecía de evaluación de actividades administrativas.

\section{Cuadro 6. Opiniones sobre las políticas y programas gubernamentales}

\begin{tabular}{|c|c|c|}
\hline \multirow{2}{*}{ Tema } & \multicolumn{2}{|l|}{ Respuestas } \\
\hline & Ejidatarios y comuneros & Funcionarios \\
\hline $\begin{array}{l}\text { Sobre las políticas } \\
\text { y programas gu- } \\
\text { bernamentales }\end{array}$ & $\begin{array}{l}\text { - Programas para el campo, mal dise- } \\
\text { ñados e inefectivos } \\
\text { - Falta de incentivos económicos para } \\
\text { la conservación con beneficios direc- } \\
\text { tos a ejidos y comunidades } \\
\text { - Falta de personal de gobierno para } \\
\text { labores de vigilancia y ejecución de } \\
\text { programas gubernamentales } \\
\text { - Falta de voluntad política de autori- } \\
\text { dades gubernamentales e ignorancia } \\
\text { de los funcionarios de necesidades y } \\
\text { dinámicas comunitarias y ejidales }\end{array}$ & $\begin{array}{l}\text { - Programas de conservación carentes de } \\
\text { una visión integral y estrategias de de- } \\
\text { sarrollo rural } \\
\text { - Falta de recursos económicos y humanos } \\
\text { - Instrumentos legales atrasados y reba- } \\
\text { sados } \\
\text { - Falta de continuidad de los programas } \\
\text { - Ineficiencia en el uso de recursos econó- } \\
\text { micos } \\
\text { - Disputas de poder entre diversas instan- } \\
\text { cias con atribuciones en el suelo de con- } \\
\text { servación }\end{array}$ \\
\hline
\end{tabular}

Fuente: elaboración propia a partir del análisis de entrevistas.

De acuerdo con la narrativa de los comuneros (en $80 \%$ de las respuestas obtenidas), los programas gubernamentales de conservación están mal diseñados y aplicados, y faltan incentivos económicos 
para la conservación, con beneficios directos a ejidos y comunidades. Mencionan como ejemplo el apoyo que las instancias gubernamentales dan a ciertos programas, como las ollas de captación en SC, en lugar de impulsar actividades productivas que, desde la perspectiva de los ejidatarios y comuneros, tendrían mayor impacto para los objetivos de conservación de dichas instancias. Un 67 $\%$ de los comuneros mencionan que falta personal de gobierno para las labores de vigilancia y para la ejecución de programas gubernamentales. Asimismo, en un $60 \%$ de las respuestas de éstos se expresa la percepción de que falta voluntad política por parte de las autoridades gubernamentales y que los funcionarios ignoran las necesidades y dinámicas comunitarias y ejidales.

\section{Opiniones sobre las consecuencias del SC}

Cerca del $93 \%$ de los funcionarios mencionan que los dueños de la tierra sufren afectaciones por las restricciones al uso de suelo. Un $67 \%$ de funcionarios mencionan que existen limitantes para proyectos productivos. El $40 \%$ de funcionarios señalan que hay cotos al desarrollo urbano y manipulación hacia los habitantes del SC (Cuadro 7). Todos los comuneros y ejidatarios mencionan la prevalencia de prohibiciones hacia actividades agrícolas y pecuarias. El 87 \% de los comuneros y ejidatarios mencionan como consecuencia la pérdida de patrimonio familiar por el decreto del SC. Los propietarios de la tierra cuestionan las restricciones en relación con el uso de los recursos naturales y las formas de vida agrícola, las cuales se han visto alteradas a partir del decreto del Programa de Ordenamiento Ecológico. Otro punto importante que se repite en las narrativas de los comuneros es que sus derechos de propiedad quedan imputados e incluso anulados, al percibir que ante las leyes que acompañan el decreto del SC, desaparece su patrimonio familiar (Cuadro 7).

\section{Cuadro 7. Opiniones sobre las consecuencias del suelo de conservación (SC)}

\begin{tabular}{|c|c|c|}
\hline \multirow{2}{*}{ Tema } & \multicolumn{2}{|l|}{ Respuestas } \\
\hline & Ejidatarios y comuneros & Funcionarios \\
\hline $\begin{array}{l}\text { Sobre las con- } \\
\text { secuencias del } \\
\text { decreto del SC }\end{array}$ & $\begin{array}{l}\text { - Prevalencia de aspectos pro- } \\
\text { hibitivos hacia actividades } \\
\text { agrícolas } \\
\text { - Pérdida del patrimonio fami- } \\
\text { liar. No es ya posible heredar } \\
\text { a los hijos un lugar para vivir }\end{array}$ & $\begin{array}{l}\text { - Hay afectación a los dueños de la tierra por las res- } \\
\text { tricciones al uso de suelo } \\
\text { - Existen limitantes a proyectos productivos } \\
\text { - Se afectan intereses inmobiliarios } \\
\text { - Hay limitantes al desarrollo urbano } \\
\text { - Hay procesos de manipulación política hacia los ha- } \\
\text { bitantes del SC }\end{array}$ \\
\hline
\end{tabular}

Fuente: elaboración propia a partir del análisis de entrevistas. 


\section{Discusión}

En este estudio se tomó la decisión de utilizar el discurso para descifrar los mecanismos de construcción social relacionados con el SC por parte de dos grupos de actores que se consideran importantes para la preservación de este territorio. Esto nos permite tener un esbozo sobre sus respectivos intereses y discutir sus límites e implicaciones. En este apartado se abordará cómo se visualiza y define el SC, cómo se trata el tema de la expansión urbana, y finalmente, la manera en que se trata el tema de las políticas relativas a la preservación del territorio.

\section{¿Cómo se comprende y define el suelo de conservación?}

La complejidad de este territorio se observa en las distintas visiones que se han expuesto, ya que para los funcionarios destaca la funcionalidad de este territorio en relación a la ciudad, mientras que para los comuneros y ejidatarios su función es la preservación de rasgos culturales y la provisión de recursos, en tanto su enfoque está en la dimensión local. En este sentido, las decisiones, según las explicaciones de los comuneros entrevistados, se toman en función de una idea preconstruida por las autoridades acerca del SC, la cual pocas veces coincide con la realidad que experimentan los habitantes de las comunidades localizadas en el área en cuestión.

Por otro lado, los comuneros, aun cuando reconocen la importancia de la preservación del bosque, flora y fauna en el SC, también afirman que es un espacio donde llevan a cabo actividades culturales y productivas de las que obtienen recursos para su subsistencia, y en las que además sustentan su identidad. Pese a que muchas de las decisiones sobre lo que sucede en el SC, según los propietarios, son tomadas por los funcionarios, dichas decisiones son cuestionadas constantemente por los comuneros y ejidatarios. Apoyándose en otros estudios, Durand (2019: 23-34) menciona: "esta situación genera restricciones para la penetración de las corporaciones y fuerza a los actores interesados en la conservación, sea el gobierno, las empresas, los académicos o las ONG, a establecer diálogos, a interactuar y negociar con las comunidades locales", ya que son los ejidatarios y comuneros mexicanos quienes aún resguardan la mayor parte de los recursos biológicos del país.

En tanto los funcionarios destacan la cualidad "verde" del SC, es decir, en tanto sostienen la idea de que éste es un espacio poco alterado que aporta importantes servicios ambientales a la ciudad, consideran como acertado que el gobierno de la local utilice éste como una categoría jurídica para limitar la expansión urbana y reservar áreas protegidas ante el cambio de uso de suelo. Se puede decir que las representaciones imbuidas en los discursos de los funcionarios enuncian imperativos conservacionistas para los distintos paisajes del SC a través de un orden binario (ciudad/naturaleza) que impone elementos tales como lo prohibido/lo permitido, así como procesos de inclusión/exclusión, en el quehacer cotidiano de la toma de decisiones.

Sociedad y Ambiente, 23, 2020, ISSN: 2007-6576, pp. 1-29. doi: 10.31840/sya.vi23.2149 
Profundizando en este aspecto, las visiones contrapuestas entre los funcionarios —que consideran que una condición sine qua non para la conservación es que los SC sean espacios intocados- y los propietarios — quienes necesitan utilizar estos territorios en actividades agrícolas-, pone de manifiesto dos formas distintas de construcción del espacio ambiental. Se puede decir que los funcionarios implícitamente tienen una concepción de conservación tipo fortaleza (Vaccaro et al., 2013), caracterizada por la aplicación de una forma de gobierno burocrático estatal (Lowe, 2006), dominado por expertos (Saberwal et al., 2001), con decisiones de Estado centralizadas y verticales (Vaccaro et al., 2013). El encuadre jurídico narrado por los funcionarios adquiere características determinadas al haber sido históricamente codificado durante la década de los ochenta con la intervención de grupos académicos ambientalistas que contribuyeron a caracterizar este territorio desde el punto de vista de diagnósticos técnicos de flora, fauna y servicios ecosistémicos (Mumme et al., 1988).

Al mismo tiempo, los ejidatarios y comuneros reconocen la existencia del SC como una medida jurídica para la preservación de ciertos espacios, sin embargo, la codificación que hacen de este territorio incluye y constituye una parte importante de su propia identidad coincidiendo con lo que menciona Durand (2019) en su estudio de conservación de la Lacandona, y Haenn (1999) en su estudio sobre Calakmul. Para ejidatarios y comuneros es una porción de la ciudad con atributos culturales diferentes al resto del espacio urbano, los cuales deben mantenerse.

\section{Las visiones sobre expansión urbana}

Los funcionarios señalan como uno de los factores que agudizan el problema de la expansión urbana, la utilización de los programas de desarrollo urbano como instrumentos para regularizar asentamientos humanos irregulares (AHI). A pesar de que el SC es reconocido por los funcionarios como una medida adecuada de política ambiental dotada de fundamentos jurídicos, mencionan que en los hechos, lo ambiental se subordina a los urbano; así por ejemplo, aun cuando están vigentes las normas del Programa General de Ordenamiento Ecológico para evitar la expansión urbana, otro instrumento de carácter administrativo, como son los Programas Delegacionales de Desarrollo Urbano, son utilizados por las autoridades para regularizar asentamientos humanos, coincidiendo con lo que mencionan Aguilar y Santos (2011a y 2011b) en cuanto a que la política urbana ha tomado posiciones tolerantes hacia la ocupación ilegal.

Por otro lado, lo dicho por ejidatarios y comuneros acerca de la promoción de asentamientos urbanos irregulares por parte de diputados y políticos reafirma en parte lo que mencionan los funcionarios. La existencia de AHI afecta la política urbana porque hay negociaciones con políticos e intercambio de favores por votos. Al final, esos favores se convierten en beneficios económicos potenciales para los invasores, a través de los servicios urbanos (agua y electricidad) que vienen a

Sociedad y Ambiente, 23, 2020, ISSN: 2007-6576, pp. 1-29. doi: 10.31840/sya.vi23.2149 
adquirir (Aguilar y Santos, 2011a y 2011b). Algunos políticos incluso alientan la ocupación ilegal de tierras públicas (Macedo, 2000), coincidiendo con lo que mencionan Ward (1998) y Varley (2004) entre otros, acerca de que la regularización de estos asentamientos informales ha servido como un mecanismo para mantener la estabilidad política en el contexto político urbano.

Esto pone de manifiesto que hay intereses políticos que buscan generar bases de apoyo que intercambian votos en los periodos de elecciones a puestos de gobierno y a diputaciones, a cambio de obtener solución para asuntos relacionados con la permanencia en los territorios, así como de lograr la dotación de servicios e infraestructura para mejorar las condiciones de vida de las colonias de escasos recursos asociadas con los asentamientos humanos irregulares ubicados en SC. En los hechos, las medidas que buscan proteger la naturaleza no se practican, quedando sobre el papel tanto en el Programa General de Ordenamiento Ecológico vigente como en los programas de manejo de las áreas naturales protegidas ubicadas en el SC.

En la práctica, el SC ha funcionado como una reserva de tierra (Pérez Campuzano et al., 2016) que permite a los grupos pobres concentrarse en asentamientos informales, lo que implica otra deficiencia de la política urbana porque, dada la falta de reservas de tierra en la Ciudad de México, no sólo es necesaria una política de redensificación orientada a los pobres, sino también una política a nivel metropolitano para encontrar áreas para canalizar el crecimiento futuro de la ciudad y los medios urbanos para hacerlo. Pero esta doble estrategia con una visión tanto local como metropolitana no existe (Aguilar y Santos, 2011a y 2011b).

\section{Las políticas y programas gubernamentales}

Los funcionarios y comuneros priorizan de forma distinta las políticas, divergiendo igualmente sus percepciones sobre las dificultades asociadas con su ejecución en el SC. Muchos de los funcionarios identifican como problema la falta de políticas agropecuarias y forestales que compitan con la especulación inmobiliaria y la venta de terrenos. En sus respuestas se hace patente su percepción acerca de que los programas de conservación carecen de una visión integral que incorpore estrategias de desarrollo rural. Otro elemento a destacar también en sus respuestas es la mención de la falta de recursos económicos y humanos, así como su visión de que los instrumentos legales están atrasados e incluso, rebasados. En la narrativa de los comuneros, gran parte de las respuestas mencionan programas gubernamentales de conservación mal diseñados y aplicados. También mencionan que hay una falta de personal de gobierno para labores de vigilancia y ejecución de programas gubernamentales. La falta de voluntad política de autoridades gubernamentales e ignorancia de los funcionarios de las necesidades y dinámicas comunitarias y ejidales, se expresa en un $60 \%$ de las respuestas.

Sociedad y Ambiente, 23, 2020, ISSN: 2007-6576, pp. 1-29. doi: 10.31840/sya.vi23.2149 


\section{Socjedad \\ Ambiente}

En una primera aproximación, se puede observar que hay coincidencias entre comuneros y funcionarios con respecto a las fallas de la política en la integración de visiones de conservación, desarrollo agrícola y contención de la expansión urbana. Sin embargo hay que señalar que a los propietarios se les hace responsables de frenar la devastación de la superficie boscosa, a la vez que se les restringe el libre uso de los paisajes agrícolas y pecuarios que son de su propiedad, condicionándoles además los apoyos económicos de los programas gubernamentales dirigidos a los proyectos productivos, a los cuales se les atribuye un doble propósito: primero, el mejoramiento de las condiciones de vida de los ejidatarios y comuneros, y segundo, ser parte de una estrategia de mejoramiento de las condiciones ambientales prevalecientes en el SC.

La deficiencia de los espacios de participación promovidos desde las instancias gubernamentales hace que el diseño de gestión integral de los programas ambientales y de desarrollo agrícola — para el cual se busca tanto la participación de las entidades de gobierno como de las autoridades ejidales y comunales - sea muy limitada. Por un lado, no evita que sean elegidas opciones que propician la especulación inmobiliaria y, por otro lado, conduce a una mayor marginación política y económica de los habitantes del SC. Pese a que varios de los instrumentos de política relacionados con el SC enuncian el respeto de la propiedad agraria ejidal y comunal y también mencionan la necesidad de la participación informada por parte de los propietarios, en los hechos resulta muy trabajoso para las instancias de gobierno construir y generar condiciones para que esto suceda, pues esto implica un quehacer cotidiano por parte de equipos promotores convencidos de su papel transformador y benefactor. Por lo tanto, mientras los programas de gobierno sigan priorizando la protección de la biodiversidad y la preservación de los terrenos con bosques característicos de la sierra del Chichinautzin sobre el bienestar de los productores agrícolas del SC, difícilmente se podrá mantener la extensión de los paisajes boscosos y agropecuarios ante el embate de la expansión urbana.

En este sentido, los programas de política que operan en el SC, reafirman la condición de perdedores de los propietarios de los terrenos boscosos del SC, mientras que los ganadores son los usufructuarios urbanos de los servicios ambientales que proporciona este territorio, lo cual se refleja, por ejemplo, en los "beneficios mutuos" de distribución y consumo de agua. En este sentido, los habitantes urbanos son ganadores, porque ni siquiera se les perturba con información acerca de la existencia de este territorio en la ciudad y su vínculo con la viabilidad ambiental de la Ciudad de México, en tanto superficie de infiltración de agua: en las zonas de alto poder adquisitivo de la ciudad se ignora por completo que el sencillo acto de abrir las llaves del agua implica para otros habitantes de la misma ciudad el despojo de sus recursos.

Sociedad y Ambiente, 23, 2020, ISSN: 2007-6576, pp. 1-29. doi: 10.31840/sya.vi23.2149 


\section{Conclusiones}

A partir del objetivo de este estudio, se puede concluir lo siguiente: el SC es un territorio de suma importancia para la viabilidad de la Ciudad de México, sin embargo, las formas en que se visualiza a través de los diferentes relatos aquí presentados, muestra que hay puntos de divergencia en la descripción de cómo se comprende y define este territorio. En cuanto a la problemática, se puede sugerir que hay coincidencia en cuanto a la manera en que se menciona a la expansión urbana. Por otro lado, dado que se ha identificado como una dificultad la falta de consenso entre las autoridades y los comuneros y ejidatarios, un punto nodal de solución para lograr la construcción de acuerdos es la consulta hacia los pueblos originarios. La promoción de asentamientos urbanos irregulares por parte de diputados y políticos, así como la utilización de los programas de desarrollo urbano como instrumentos para regularizar asentamientos humanos irregulares (AHI), confirma lo que mencionan Ward (1998), Varley (2004) y Aguilar y Santos (2011a y 2011b) entre otros, acerca de que la regularización de estos asentamientos informales ha servido como un mecanismo para mantener la estabilidad política en el contexto político urbano.

El SC es un híbrido con espacios dinámicos y en constante transformación que tiene una parte física, una parte de prácticas materiales y mercantilizadas, así como dimensiones discursivas construidas de forma diferenciada. Concebir al SC como un territorio de interacción entre lo urbano, lo rural y lo ambiental en la periferia de la ciudad, implica que la dimensión política del espacio no sólo comprende una dimensión material, sino además dimensiones de representación en las que diversos factores intervienen en su producción. Tanto para los comuneros como para los funcionarios, los beneficiarios del SC son todos los habitantes de la ciudad, puesto que reciben agua, aire limpio, posibilidades de esparcimiento y productos agrícolas, entre muchos otros bienes y servicios. Sin embargo, el mantenimiento de estos beneficios para la ciudad implica una serie de restricciones de acceso a los recursos biológicos impuestas a través de la normatividad ambiental, que como los funcionarios reconocen, constituyen costos de conservación que son asignados y atribuidos a los ejidatarios y comuneros.

Mientras las necesidades de la gente de escasos recursos que habita en la periferia no sean atendidas de manera satisfactoria, sobre todo en relación a la vivienda, las políticas de conservación serán un compendio de buenas intenciones. Los conflictos de interpretación del SC, además de ser producto de tensiones entre visiones e intereses contrapuestos frente al uso y apropiación de un espacio particular en el que se disputa su reconocimiento y hegemonía, se gestan en un escenario donde se entremezclan y construyen simultáneamente las diferentes dimensiones de la periferia urbana, ya que son el marco en el que circulan e interactúan distintos discursos y recursos de poder. La perspectiva de este estudio puede contribuir a visibilizar esta dinámica generalizada 
también en las periferias urbanas de otras ciudades latinoamericanas, donde entran en tensión distintos actores que interactúan en territorios con procesos de expansión urbana y cambio en el uso de la tierra, en relación con el nuevo enfoque político en la gestión ambiental que incluye la centralidad de los servicios ecosistémicos.

\section{Referencias}

Aguilar, Adrián Guillermo (2008). "Peri-urbanization, Illegal Settlements, and Environmental Impact in Mexico City”. Cities, 25(3), pp. 133-145.

Aguilar, Adrián Guillermo y Santos, Clemencia (2011a). "El manejo de asentamientos humanos irregulares en el suelo de conservación del Distrito Federal. Una política urbana ineficaz". En Adrián Guillermo Aguilar e Irma Escamilla (eds.), Periurbanización y sustentabilidad en grandes ciudades. México: Cámara de Diputados/UNAM-Instituto de Geografía/CONACYT/ Miguel Ángel Porrúa, pp. 277-315.

Aguilar, Adrián Guillermo y Santos, Clemencia (2011b). "Informal Settlements' Needs and Environmental Conservation in Mexico City: An Unsolved Challenge for Land-Use Policy". Land Use Policy, 28(4), pp. 649-662.

Angelo, Hillary y Wachsmuth, David (2014). "Urbanizing Urban Political Ecology: A Critique of Methodological Cityism". International Journal of Urban and Regional Research, 39(1), pp. 16-27. Recuperado de https://doi.org/10.1111/1468-2427.12105

Allen, Adriana (2003). "Environmental Planning and Management of the Peri-urban Interface: Perspectives on an Emerging Field”. Environment and Urbanization, 15(1), pp. 135-148.

Barnaud, Cécile; Corbera, Esteve; Muradian, Roldan; Salliou, Nicolas; Sirami, Clélia; Vialatte, Aude; Choisis, Jean-Philippe; Dendoncker, Nicolas; Mathevet, Raphaël; Moreau, Clémence; Reyes-García, Victoria; Boada, Marti; Deconchat, Marc; Cibien, Catherine; Garnier, Stéphane; Maneja, Roser, y Antona, Martine (2018). "Ecosystem Services, Social Interdependencies and Collective Action: A Conceptual Framework". Ecology and Society, 23(1), 15. Recuperado de https://doi.org/10.5751/ES-09848-230115

Baró, Francesc; Chaparro, Lydia; Gómez-Baggethun, Erik; Langemeyer, Johannes; Nowak, David J., y Terradas, Jaume (2014). "Contribution of Ecosystem Services to Air Quality and Climate Change Mitigation Policies: The Case of Urban Forests in Barcelona, Spain”. Ambio, 43(4), pp. 466-479.

Barsky, Andrés (2005). "El periurbano productivo, un espacio en constante transformación. Introducción al estado del debate, con referencias al caso de Buenos Aires". Scripta Nova, revista electrónica de Geografía y Ciencias Sociales, IX(194), pp. 36. Recuperado de http://www. ub.edu/geocrit/sn/sn-194-36.htm

Bayón, María Cristina (2016). "La ciudad de los márgenes: periferias lejanas, desventajas y fragmentación social”. En Lucía Álvarez Enríquez, Gian Carlo Delgado Ramos y Alejandra Leal

Sociedad y Ambiente, 23, 2020, ISSN: 2007-6576, pp. 1-29. doi: 10.31840/sya.vi23.2149 
Martínez (coords.), Los desafíos de la ciudad del siglo XXI. México: Senado de la República, LXIII Legislatura, UNAM-CEIICH-PUEC-PUES, $562 \mathrm{pp}$.

Bischoping, Katherine y Gazso, Amber (2016). Analyzing Talk in the Social Sciences: Narrative, Conversation and Discourse Strategies. Londres: SAGE, 240 pp.

Brownlow, Alec (2006). "Inherited Fragmentations and Narratives of Environmental Control in the Intrepreneurial Philadelphia". En Nik Heynen, Maria Kaika y Erik Swyngedouw (eds.), In the Nature of Cities: Urban Political Ecology and the Politics of Urban Metabolism. Abingdon: Routledge, pp. 208-225.

Bryant, Raymond y Bailey, Sinéad (1997). Third World Political Ecology. Londres: Routledge, 237 pp.

Buchanan, Karen S. (2013). "Contested Discourses, Knowledge, and Socio-Environmental Conflict in Ecuador". Environmental Science \& Policy, 30, pp. 19-25. Recuperado de https://doi. org/10.1016/j.envsci. 2019.12.012.

Castelán, J. Enrique y Mejía, Adolfo (2011). "Política ambiental en el Suelo de Conservación del Distrito Federal". En Adrián Guillermo Aguilar e Irma Escamilla (eds.), Periurbanización y sustentabilidad en grandes ciudades. México: Cámara de Diputados, UNAM-Instituto de Geografía/CONACYT/Miguel Ángel Porrúa, pp. 253-275.

Castree, Noel y Braun, Bruce (eds.) (2001). Social Nature. Theory, Practice, and Politics. Malden, Massachusetts: Blackwell, 249 pp.

Cook, Ian y Swyngedouw, Erik (2012) "Cities, Social Cohesion and the Environment: Towards a Future Research Agenda”. Urban Studies, 49(9), pp. 1938-1958.

Crowe, Sarah; Cresswell, Kathrin; Robertson, Ann; Huby, Guro; Avery, Anthony, y Sheikh, Aziz (2011). "The Case Study Approach”. BMC Medical Research Methodology, 11(1), 100. Recuperado de https://doi.org/10.1186/1471- 2288-11-100.

Da Gamma Torres, Haroldo (2008). "Social and Environmental Aspects of Peri-urban Growth in Latin American Megacities". United Nations Expert Group Meeting on Population Distribution, Urbanization, Internal Migration and Development, pp. 21-23.

Darling, Eliza (2006). "Nature's Carnival: The Ecology of Pleasure at Coney Island". En Nik Heynen, Maria Kaika y Erik Swyngedouw (eds.), In the Nature of Cities: Urban Political Ecology and the Politics of Urban Metabolism. Abingdon: Routledge, pp. 75-92.

DDF (Departamento del Distrito Federal) (1982). Plan de Desarrollo Urbano: Distrito Federal. México, D.F.

Depietri, Yaella; Welle, Torsten, y Renaud, Fabrice G. (2013). "Social Vulnerability Assessment of the Cologne Urban Area (Germany) to Heat Waves: Links to Ecosystem Services". International Journal of Disaster Risk Reduction, 6, pp. 98-117.

Diario Oficial de la Federación (1982). "Reglamento de Zonificación", 20 de abril de 1982.

Durand, Leticia (2019). "Power, Identity and Biodiversity Conservation in the Montes Azules Biosphere Reserve, Chiapas, Mexico". Journal of Political Ecology, 26(1), pp. 19-37. 
Elmqvist, Thomas; Fragkias, Michail; Goodness, Julie; Güneralp, Burak; Marcotullio, Peter J.; McDonald, Robert I.; Parnell, Susan; Schewenius, Maria; Sendstad, Marte; Seto, Karen C., y Wilkinson, Cathy (eds.) (2013). Urbanization, Biodiversity and Ecosystem Services: Challenges and Opportunities. Dordrecht, Holanda: Springer, 755 pp.

Escandón Calderón, Jorge (2014). "Ecología política urbana y suelo de conservación en la Ciudad de México: Tlalpan como estudio de caso" (Tesis de doctorado en Ciencias Políticas y Sociales con orientación en Ciencia Política). México: Universidad Nacional Autónoma de México. Ciudad de México, 275 pp.

Escobar, Arturo (1996). "Construction Nature: Elements for a Post-structuralist Political Ecology". Futures, 28(4), pp. 325-343. Recuperado de https://doi.org/10.1016/0016-3287(96)00011-0.

Evalúa D.F. (2011). "Índice de desarrollo social de las unidades territoriales del Distrito Federal". Consejo de Evaluación del Desarrollo Social del Distrito Federal, México.

Fisher, Tania (2003). "Differentiation of Growth Process in the Periurban Region: An Australian Case Study". Urban Studies, 40(3), pp. 551-565.

Flitner, Michael (2008). "Politische Ökologie und Umweltgerechtigkeit: Konflikte um Fluglärm”. Geographische Rundschau, 60(12), pp. 50-56.

Foucault, Michel (1970). La arqueología del saber. Segunda edición. Madrid: Siglo XXI, 276 pp.

Foucault, Michel (2007). Security, Territory, Population: Lectures at the Collège de France 19771978. Nueva York: Palgrave McMillan, 417 pp.

GDF (Gobierno del Distrito Federal) (2014). "Ley de Desarrollo Urbano del Distrito Federal". Recuperado de http://aldf.gob.mx/archivo-3a6419f3c20189c5c79382d35f87c41f.pdf

Gandy, Matthew (2004). "Rethinking Urban Metabolism: Water, Space and the Modern City". City, 8(3), pp. 363-379.

Gandy, Matthew (2006). "Urban Nature and the Ecological Imaginary". En Nik Heynen, Maria Kaika y Erik Swyngedouw (eds.), In the Nature of Cities: Urban Political Ecology and the Politics of Urban Metabolism. Abingdon: Routledge, pp. 62-72.

Graham, Stephen (2006). "Urban Metabolism as Target: Contemporary War as Forced Demodernization". En Nik Heynen, Maria Kaika y Erik Swyngedouw (eds.), In the Nature of Cities: Urban Political Ecology and the Politics of Urban Metabolism. Abingdon: Routledge, pp. 245254.

Haase, Dagmar; Schwarz, Nina; Strohbach, Michael; Kroll, Franziska, y Seppelt, Ralf (2012). "Synergies, Trade-Offs, and Losses of Ecosystem Services in Urban Regions: An Integrated Multiscale Framework Applied to the Leipzig-Halle Region, Germany". Ecology and Society, 17(3): 22 .

Haenn, Nora (1999). "The Power of Environmental Knowledge: Ethnoecology and Environmental Conflicts in Mexican Conservation”. Human Ecology, 27(3), pp. 477-491.

Hajer, Maarten (1995). "Ecological Modernization as Cultural Politics". En Mike Featherstone (ed.), Theory, Culture \& Society. Teesside: University of Teesside, pp. 246-269. 
Heynen, Nik (2006a). "Green Urban Political Ecologies: Toward a Better Understanding of Inner-City Environmental Change". Environment and Planning A: Economy and Space, 38(3), pp. 499-516.

Heynen, Nik (2006b). "Justice of Eating in the City: The Political Ecology of Urban Hunger". En Nik Heynen, Maria Kaika y Erik Swyngedouw (eds.), In the Nature of Cities: Urban Political Ecology and the Politics of Urban Metabolism. Abingdon: Routledge, pp. 120-136.

Heynen, Nik; Kaika, Maria, y Swyngedouw, Erik (2006). "Urban Political Ecology: Politicizing the Production of Urban Natures". En Nik Heynen, Maria Kaika y Erik Swyngedouw (eds.), In the Nature of Cities: Urban Political Ecology and the Politics of Urban Metabolism. Abingdon: Routledge, pp. 1-20.

Hornis, Willemieke y Eck, Jan Ritsema van (2008). "A Typology of Peri-urban Areas in the Netherlands". Tijdschrift voor Economische en Sociale Geografie, 99(5), pp. 619-628.

Huang, Shu-Li; Wang, Szu-Hua, y Budd, William W. (2009). "Sprawl in Taipei's Peri-urban Zone: Responses to Spatial Planning and Implications for Adapting Global Environmental Change". Landscape and Urban Planning, 90(1-2), pp. 20-32.

INEGI (Instituto Nacional de Estadística y Geografía) (2006). "Banco de Información Económica. Series no actualizadas". Recuperado de www.inegi.org.mx

INEGI (2010). "Banco de Información Económica /PIB por entidad federativa". Recuperado de www.inegi.org.mx

Kaika, Maria (2006). "The Political Ecology of Water Scarcity: The 1989-1991 Athenian Drought". En Nik Heynen, Maria Kaika y Erik Swyngedouw (eds.), In the Nature of Cities: Urban Political Ecology and the Politics of Urban Metabolism. Abingdon: Routledge, pp. 157-172.

Keil, A., D'souza A. y McDonald A. (2016). "Sustainable wheat intensification in the eastern Indo-Gangetic Plains: CSISA's approach to scaling zero-tillage wheat in Bihar". CIMMYT.

Keil, Roger y Boudreau, Julie-Anne (2006). "Metropolitics and Metabolics: Rolling out Environmentalism in Toronto". En Nik Heynen, Maria Kaika y Erik Swyngedouw (eds.), In the Nature of Cities: Urban Political Ecology and the Politics of Urban Metabolism. Abingdon: Routledge, pp. 41-62.

Keil, Roger (ed.) (2013). Suburban Constellations: Governance, Land and Infrastructure in the 21st Century. Berlín: Jovis, 208 pp.

Keivani, Ramin y Mattingly, Michael (2007). "The Interface of Globalization and Peripheral Land in the Cities of the South: Implications for Urban Governance and Local Economic Development". International Journal of Urban and Regional Research, 31(2), pp. 459-474.

Kirby, Sandra y McKenna, Kate (1989). Experience. Research. Social Change: Methods from the Margins. Toronto: Garamond Press, 184 pp.

Kritsanaphan, Amorn y Sajor, Edsel (2011). "Intermediaries and Informal Interactions in Decentralised Environmental Management in Peri-urban Bangkok". International Development Planning Review, 33(3), pp. 247-271. 
Larondelle, Neele; Haase, Dagmar, y Kabisch, Nadja, (2014). "Mapping the Diversity of Regulating Ecosystem Services in European Cities". Global Environmental Change, 26, pp. 119-129.

Latour, Bruno (1993). We Have Never Been Modern. Cambridge: Mass, 168 pp.

Latour, Bruno (1998) "To Modernise or to Ecologise? That is the Question". En Bruce Braun y Noel Castree (eds.), Remaking Reality. Nature at the Millenium. Londres y Nueva York: Routledge, pp. 221-242.

Latour, Bruno (2004). Politics of Nature. How to Bring the Sciences into Democracy. Cambridge, Massachusetts: Harvard University Press, 307 pp.

Lerner, Amy M. y Eakin, Hallie (2011). "An Obsolete Dichotomy? Rethinking the Rural-Urban Interface in Terms of Food Security and Production in the Global South". The Geographical Journal, 177(4), pp. 311-320. doi: http://dx.doi.org/10.1111/j.1475-4959.2010.00394.x

Liu Yanfang, Ming Dongping y Yang Jianyu (2002). "Optimization of land use structure based on ecological GREEN equivalent", Geo-spatial Information Science, 5:4, 60-67, doi: 10.1007/ BF02826478

Llanos, Douglas y Almandoz, Arturo (2008). “Aplicación del modelo de Cinturón Verde en la Ciudad de Caracas, Venezuela”. Provincia, 19, pp. 143-160.

Loftus, Alex (2006). "The Metabolic Processes of Capital Accumulation in Durban's Waterscape". En Nik Heynen, Maria Kaika y Erik Swyngedouw (eds.), In the Nature of Cities: Urban Political Ecology and the Politics of Urban Metabolism. Abingdon: Routledge, pp. 173-190.

Lowe, Celia (2006). Wild Profusion: Biodiversity Conservation in an Indonesian Archipelago. Princeton: Princeton University Press, 224 pp.

Macedo, Joseli (2000). "Land Use Policies and Urbanization of Informal Settlements: Planning Initiatives for Environmental Protection Areas in Curitiba, Brazil" (Dissertation for the degree of Doctor of Philosophy). Florida, EUA: State University System of Florida, 220 pp.

Marvin, Simon y Medd, Will (2006). "Metabolisms of the Obe-City. Flows of Fat through Bodies, Cities and Sewers". En Nik Heynen, Maria Kaika y Erik Swyngedouw (eds.), In the Nature of Cities: Urban Political Ecology and the Politics of Urban Metabolism. Abingdon: Routledge, pp. 143-156.

MEA (Millenium Ecosystem Assesment) (2005). Ecosystems and Human Well-being: Current State and Trends. Washington, DC: Island Press, 948 pp.

Müller-Mahn, Detlef (2006). "Wasserkonflikte im Nahen Osten - eine Machtfrage". Geographische Rundschau, 58(2), pp. 40-48.

Mumme, Stephen P.; Bath, C. Richard, y Assetto, Valerie J. (1988). "Political Development and Environmental Policy in Mexico". Latin American Research Review, 23(1), pp. 7-34.

Myers, Garth Andrew (2008). "Peri-urban Land Reform, Political-Economic Reform, and Urban Political Ecology in Zanzibar". Urban Geography, 29(3), pp. 264-288. doi: 10.2747/0272 3638.29.3.264 
Nechyba, Thomas J., and Randall P. Walsh. "Urban sprawl." Journal of economic perspectives 18.4 (2004): 177-200.

Nelson, Lise y Nelson, Peter B. (2010). "The Global Rural: Gentrification and Linked Migration in the Rural USA". Progress in Human Geography, 35(4), pp. 441-459.

Oliver, Stuart (2006). “The Desire to Metabolize Nature”. En Nik Heynen, Maria Kaika y Erik Swyngedouw (eds.), In the Nature of Cities: Urban Political Ecology and the Politics of Urban Metabolism. Abingdon: Routledge, pp. 93-109.

PAOT (Procuraduría Ambiental y del Ordenamiento Territorial) (2009). "Estudio sobre la superficie ocupada en Áreas Naturales Protegidas del Distrito Federal”. Recuperado de http://centro.paot.org.mx/documentos/paot/estudios/EOT-03-2009.pdf

Pelling, Mark (2003). "Toward a Political Ecology of Urban Environmental Risk. The Case of Guyana”. En Karl S. Zimmerer y Thomas J. Bassett (eds.), Political Ecology. An Integrative Approach to Geography and Environment-Development Studies. Nueva York y Londres: Guilford Press, pp. 73-93.

Pellow, David N. (2006) "Transnational Alliances and Global Politics: New Geographies of Urban Environmental Justice Struggles". En Nik Heynen, Maria Kaika y Erik Swyngedouw (eds.), In the Nature of Cities: Urban Political Ecology and the Politics of Urban Metabolism. Abingdon: Routledge, pp. 226-244.

Perevochtchikova, María y Vázquez, Adrián (2012). “The Federal Program of Payment for Hydrological Environmental Services as an Alternative Instrument for Integrated Water Resources Management in Mexico City". The Open Geography Journal, 5, pp. 35-46.

Pérez-Campuzano, Enrique (2011). "Periferia urbana e incentivos económicos para la conservación ambiental". Regions and Cohesion, 1(2), pp. 78-103.

Pérez-Campuzano, Enrique; Perevochtchikova, María, y Ávila-Foucat, V. Sophie (coords.) (2011). Suelo de conservación del Distrito Federal. Hacia una gestión y manejo sustentable. México: Miguel Ángel Porrúa/Instituto Politécnico Nacional, 246 pp.

Pérez-Campuzano, Enrique; Perevochtchikova, María, y Ávila-Foucat, V. Sophie (2012). ¿Hacia un manejo sustentable del suelo de conservación del Distrito Federal? México: Miguel Ángel Porrúa/Instituto Politécnico Nacional.

Pérez-Campuzano, Enrique; Avila-Foucat, V. Sophie, y Perevochtchikova, María (2016). "Environmental Policies in the Peri-Urban Area of Mexico City: The Perceived Effects of Three Environmental Programs". Cities, 50, pp. 129-136.

Pezzoli, Keith (1998). Human Settlements and Planning for Ecological Sustainability: The Case of Mexico City. Massachusetts: MIT Press, 464 pp.

Portnov, Boris A. y Pearlmutter, David (1999). "Sustainable Urban Growth in Peripheral Areas". Progress in Planning, 52(4), pp. 239-308. 
Ravetz, Joe; Fertner, Christian, y Nielsen, Thomas Sick (2013). “The Dynamics of Peri-urbanization”. En Kjell Nilsson et al. (eds.), Peri-urban Futures: Scenarios and Models for Land Use Change in Europe. Berlín, Heidelberg: Springer-Verlag, 453 pp.

Rikoon, J. Sanford (2006). "Wild Horses and the Political Ecology of Nature Restoration in the Missouri Ozarks". Geoforum, 37, pp. 200-211. Recuperado de https://doi.org/10.1016/j.geoforum.2005.01.010

Robbins, Paul (2004). Political Ecology: A Critical Introduction. Malden: MA Blackwell, 242 pp.

Robbins, Paul y Sharp, Julie (2006). "Turfgrass Subjects: The Political Economy of Urban Monoculture”. En Nik Heynen, Maria Kaika y Erik Swyngedouw (eds.), In the Nature of Cities: Urban Political Ecology and the Politics of Urban Metabolism. Abingdon: Routledge, pp. 110-128.

Robbins, Paul (2007). Lawn People: How Grasses, Weeds and Chemicals Make us Who we Are. Philadelphia, PA: Temple University Press, 186 pp.

Ruiz-Rivera, Naxhelli y Delgado Campos, Javier (2008). "Territorio y nuevas ruralidades: un recorrido teórico sobre las transformaciones de la relación campo-ciudad”. EURE, XXXIV(102), pp. 77-95.

Saavedra, Zenia; Ojeda Revah, Lina, y López Barrera, Faustino (2011). "Identification of Threatened Areas of Environmental Value in the Conservation Area of Mexico City, and Setting Priorities for their Protection”. Investigaciones Geográficas, 74, pp. 19-34.

Saberwal, Vasant; Rangarajan, Mahesh, y Kothari, Ashish (2001). People, Parks, and Wildlife: Towards Coexistence. Nueva Delhi: Orient, 143 pp.

Schteingart, Martha (2016). "Infraestructura y división del espacio en las ciudades”. En Lucía Álvarez Enríquez, Gian Carlo Delgado Ramos, Alejandra Leal Martínez (coords.), Los desafíos de la ciudad del siglo XXI. México: Senado de la República, LXIII Legislatura/ UNAM-CEIICH-PUEC-PUES, 562 pp.

Seto, Karen C.; Dhakal, Shobhakar; Bigio, Anthony; Blanco, Hilda; Delgado, Gian Carlo; Dewar, David; Huang, Luxin; Inaba, Atsushi; Kansal, Arun; Lwasa, Shuaib; McMahon, James; Müller, Daniel B.; Murakami, Jin; Nagendra, Harini, y Ramaswami, Anu (2014). "Human Settlements, Infrastructure and Spatial Planning”. En Ottmar Edenhofer, Ramón Pichs-Madruga, Youba Sokona, Ellie Farahani, Susanne Kadner, Kristin Seyboth, Anna Adler, Ina Baum, Steffen Brunner, Patrick Eickemeier, Benjamin Kriemann, Jussi Savolainen, Steffen Schlömer, Christoph von Stechow, Timm Zwickel y Jan C. Minx (eds.) (2014) Climate Change 2014: Mitigation of Climate Change. Contribution of Working Group III to the Fifth Assessment Report of the Intergovernmental Panel on Climate Change. Reino Unido y EUA: Cambridge University Press.

Sheinbaum-Pardo Claudia y Escandón-Calderón, Jorge Alberto (2008). "El Suelo de Conservación”. En La problemática ambiental de la Ciudad de México. Diagnóstico y experiencias de gestión. México: Limusa/Instituto de Ingeniería, 309 pp.

Sheinbaum-Pardo, Claudia (2011). "La compleja problemática del Suelo de Conservación del Distrito Federal: Apuntes para su conservación”. En Enrique Pérez Campuzano, Maria Pere- 
vochtchikova y V. Sophie Ávila Foucat (coords.), Suelo de Conservación del Distrito Federal. Hacia una gestión y manejo sustentable. México: Instituto Politécnico Nacional/Miguel Ángel Porrúa, pp. 13-37.

Simon, David (2008). "Urban Environments: Issues on the Peri-urban Fringe". Annual Review of Environment and Resources, 33, pp. 167-185.

Simonian, Lane (1999). La defensa de la tierra del jaguar. Una historia de la conservación en México. México: INE-SEMARNAP, $352 \mathrm{pp}$.

Smith Laila y Ruiters, Greg (2006). "The Public/Private Conundrum of Urban Water: A View from South Africa". En Nik Heynen, Maria Kaika y Erik Swyngedouw (eds.), In the Nature of Cities: Urban Political Ecology and the Politics of Urban Metabolism. Abingdon: Routledge, pp. 191-207.

Snow, David A. y Benford, Robert D. (1988). "Ideology, Frame Resonance, and Participant Mobilization”. International Social Movement Research, 1(1), pp. 197-217.

Swyngedouw, Erik (1996). "The City as a Hybrid - On Nature, Society and Cyborg Urbanization". Capitalism, Nature, Socialism, 7(2), pp. 65-80.

Swyngedouw, Erik (2006a). "Circulations and Metabolisms: (Hybrid) Natures and (Cyborg) Cities”. Science as Culture, 15(2), pp. 105-122. doi:10.1080/09505430600707970

Swyngedouw, Erik (2006b). "Metabolic Urbanization. The Making of Cyborg Cities". En Nik Heynen, Maria Kaika y Erik Swyngedouw (eds.), In the Nature of Cities: Urban Political Ecology and the Politics of Urban Metabolism. Abingdon: Routledge, pp. 21-40.

Swyngedouw, Erik (2015). "Urbanization and Environmental Futures: Politicizing Urban Political Ecologies". En Tom Perreault, Gavin Bridge y James McCarthy (eds.), The Routledge Handbook of Political Ecology. Londres y Nueva York: Taylor \& Francis Group, 646 pp.

Taylor, Laura (2011). "No Boundaries: Exurbia and the Study of Contemporary Urban Dispersion". GeoJournal, 76(4), pp. 323-339.

UN DESA (United Nations Department of Economic and Social Affairs) (2013). "World Population Prospects: The 2012 Revision". Recuperado de https://population.un.org/wpp/Publications/Files/WPP2012_HIGHLIGHTS.pdf

UN DESA (2014). “World Urbanization Prospects: The 2014 Revision”. Recuperado de https://population.un.org/wup/Publications/Files/WUP2014-Methodology.pdf

Vaccaro, Ismael; Beltran, Oriol, y Paquet, Pierre Alexandre (2013). "Political Ecology and Conservation Policies: Some Theoretical Genealogies". Journal of Political Ecology, 20(20), pp. $255-272$.

Varley, A. (2004) "Los usos políticos de la ilegalidad: La evidencia del México urbano". En E. Fernandes y A. Varley, Ciudades ilegales. La ley y el urbanismo en países en vías de desarrollo. Cochabamba, Bolivia: Promesha, pp. 209-230.

Vejre, Henrik, Jens Peter Vesterager, Lone S. Kristensen y Jørgen Primdahl (2001). "Stakeholder and expert-guided scenarios for agriculture and landscape development in a groundwater 
protection area." Journal of Environmental Planning and Management 54.9, pp. 1169-1187. doi: 10.1080/09640568.2011.560782

Veron, René (2006) "Remaking Urban Environments: The Political Ecology of Air Pollution in Delhi”. Environment and Planning A, 38(11), pp. 2093-2109. doi: 10.1068/a37449

Vitz, Matthew (2012). "La ciudad y sus bosques. La conservación forestal y los campesinos en el valle de México, 1900-1950”. Estudios de Historia Moderna y Contemporánea de México, 43, pp. 135-172.

Von Bertrab Tamm, Alejandro Ignacio (2013). "De tilapias y ajolotes: Discursos sobre la restauración ecológica en el lago de Xochimilco" (Tesis de Doctorado en Ciencias Políticas y Sociales). México: Universidad Autónoma de México. Ciudad de México, 248 pp. Recuperado de http://132.248.9.195/ptd2013/agosto/0698408/0698408.pdf

Ward, Peter. M. (1999) Colonias and public policy in Texas and Mexico: Urbanization by stealth. The University of Texas Press, Austin.

Zimmer, Anna (2010). "Urban Political Ecology: Theoretical Concepts, Challenges, and Suggested Future Directions”. Erdkunde, 64(4), pp. 343-354.

Editor: Cristian Kraker Castañeda Recibido: 16 de agosto de 2019 Aceptado: 11 de marzo de 2020 